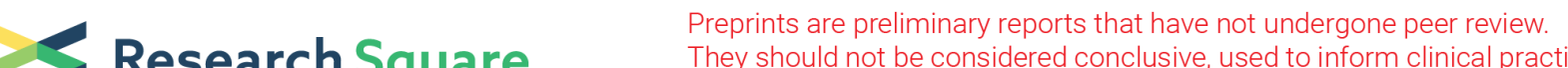 $\begin{array}{ll}\text { Research Square } & \text { They should not be considered conclusive, used to inform clinical practice, } \\ \text { or referenced by the media as validated information. }\end{array}$
}

\section{Prognostic Value of Immune-Related IncRNA Pairs In Patients with Bladder Cancer}

\section{zhenzhen Gao}

The Second Affiliated Hospital of Jiaxing University

\section{Dongjuan Wu}

Jiaxing second hospital: The Second Affiliated Hospital of Jiaxing University

\section{Wenwen Zheng}

Jiaxing second hospital: The Second Affiliated Hospital of Jiaxing University

\section{taohong Zhu}

The Second Affiliated Hospital of Jiaxing University

\section{Ting Sun}

Nanhu District central hospital

\section{Lianhong Yuan}

Nanhu District central hospital

\section{Faming Fei}

The Second Affiliated Hospital of Jiaxing University

\section{Peng Fu ( $\sim$ fupeng24@163.com )}

The Second Affiliated Hospital of Jiaxing University https://orcid.org/0000-0003-2982-6300

\section{Research}

Keywords: bladder cancer, immunotherapy, long non-coding RNA, risk model, prognosis

Posted Date: May 17th, 2021

DOI: https://doi.org/10.21203/rs.3.rs-517245/v1

License: (c) (1) This work is licensed under a Creative Commons Attribution 4.0 International License. Read Full License 


\section{Abstract}

Background: The characteristics of immune-related long non-coding ribonucleic acids (ir-lncRNAs), regardless of their specific expression level, have important implications for the prognosis of patients with bladder cancer. Methods: Based on The Cancer Genome Atlas (TCGA) database, we downloaded original transcript data, obtained the ir-IncRNAs using a coexpression method, and identified the differentially expressed pairs of ir-IncRNAs (DE-ir-IncRNAs) using univariate analysis. The IncRNA pairs were verified using a Lasso regression test. Thereafter, receiver operating characteristic curves (ROC) were generated; the area under the curve was calculated; the Akaike information criterion (AIC) of the 5-y ROC was determined; the optimal cutoff value of the high- and low-risk populations of patients with bladder cancer was confirmed, and the optimal risk model was established. The clinical value of the model was verified using survival analysis, clinicopathological characteristics, presence of tumor-infiltrating immune cells, and chemotherapy efficacy evaluation.

Results: In total, 49 pairs of DE-ir-IncRNAs were identified, and 21 pairs were included in the Cox regression model. In this study, ir-IncRNA pairs were obtained, and a risk regression model was established on the premise of not involving the specific expression value of transcripts.

Conclusions: The method and model used in this study have important clinical predictive value for bladder cancer and other malignant tumors.

\section{Background}

The incidence and mortality of bladder cancer (BC) are reported to be approximately 500,000 and 200,000 worldwide, respectively, in $2020^{[1]}$. Bladder cancer is known to include two subtypes: noninvasive and muscle-invasive bladder cancer. Non-muscle-invasive bladder cancer (NMIBC) is the predominant subtype with a high incidence of recurrence, whereas muscle-invasive bladder cancer (MIBC) accounts for approximately $25 \%$ of patients with this disease ${ }^{[2]}$. Bacillus Calmette-Guérin (BCG), a type of Mycobacterium, was developed as an antituberculosis vaccine. In particular, BCG has been reported to prevent recurrence in patients with NMIBC, with the majority progressing to the MIBC subtype. Systemic chemotherapy has been the standard first-line therapy for MIBC. Patients who have failed first-line treatments have few second-line chemotherapeutic options ${ }^{[3]}$, including gemcitabine, paclitaxel, and ifosfamide, which have been associated with a restricted clinical benefit. With the advent of immune checkpoint inhibitors (ICls), patients from KEYNOTE045 ${ }^{[4]}$ treated with pembrolizumab as second-line therapy were reported to have an approximately 10.3-month survival, with tumor response (objective response rate) in the pembrolizumab group of $21.1 \%$, greater than that in the chemotherapy group (11\%). After that, ICls (atezolizumab and pembrolizumab) were confirmed to be effective as first-line therapy, based on the results of two phase II clinical trial studies, including NCT02108652 ${ }^{[5]}$ and KEYNOTE052 ${ }^{[6]}$. Herein, both the European Medicines Agency (EMA) and the Food and Drug Agency (FDA) approved atezolizumab and pembrolizumab as first-line treatment for metastatic cisplatin-ineligible MIBC, restricted to cisplatin-unfit patients with PD-L1-high tumors.. Although PD-L1 was a predictor of 
efficacy ${ }^{[7]}$, other useful biomarkers related to the ICls for patients with $\mathrm{BC}$ need to be further explored to guide clinical practice.

Long non-coding RNAs (IncRNAs), with a transcript length of more than 200 nucleotides $^{[8]}$, do not encode proteins; however, they are abundant, occupying more than 80 human transcripts. With the development of advanced scientific methods, IncRNAs have been found to be significant regulators of organic biological processes, including normal development and tumorigenesis. Urothelial carcinoma-associated IncRNA (UCA1) ${ }^{[9]}$, modulating miR-196a-5p through the regulation of CREB to accommodate cisplatin sensitivity, has been overexpressed in bladder cancer compared with other normal tissues. Some IncRNAs have been reported to regulate the tumor microenvironment by targeting genes implicated in the function of immune cells ${ }^{[10-12]}$. Moreover, some immune-related IncRNA (ir-IncRNA) signatures have been recently identified in bladder cancer, whose expression was associated with the survival of patients with $\mathrm{BC}^{[13-15]}$. However, all these prognostic models were established based on the expression of IncRNA. In our study, we established a new model to predict the efficacy of immunotherapy regardless of expression.

Previous studies have verified that combinations of two genetic markers are more accurate than single genes in establishing prognostic models for cancers. To date, few studies have confirmed the prognostic value of IncRNAs in this setting ${ }^{[16]}$. A neoteric algorithm, pairing, and iteration method was applied to construct an ir-IncRNA signature, with no need for any specific expression level. Finally, we evaluated its predictive and prognostic value in patients with MIBC.

\section{Methods}

RNA-seq data from the TCGA-BLCA project, downloaded from The Cancer Genome Atlas Program (TCGA), were integrated into fragments per kilobase million (FPKM), and the GTF files were used to annotate and differentiate mRNAs and IncRNAs downloaded from the Ensembl database (http://asia.ensembl.org). The ImmPort portal database (http://www.immport.org) was screened to acquire a confirmed immunerelated gene (ir-genes) list as well as immune-related IncRNAs (ir-InRNAs) through coexpression analysis. The relationship between ir-genes and all IncRNAs was verified by correlation tests; the highly correlated IncRNAs were considered ir-IncRNAs, with the cutoff value of correlation efficacy being more than 0.5 , and the P-value was $\leq 0.05$. Ultimately, the R package "limma" (Bioconductor, USA) was used to detect differentially expressed IncRNAs (DE-IncRNAs), with the thresholds being defined as log fold change (FC) $>2$, with a false discovery rate (FDR) less than 0.05 .

For DE-ir-IncRNA pairing, if one of two markers was highly expressed in a sample, then the sample was regarded as a highly expressing sample of the two DE-ir-IncRNA markers. DE-ir-IncRNAs were tautologically paired, and a 0 or 1 matrix was constructed. Given that $A$ is equal to IncRNA $B$ plus IncRNA $C, A$ is 1 if the expression level of IncRNA $B$ is higher than IncRNA C; if not, $A$ is defined as 0 . The established matrix was filtered. Pairs were considered to not be related to prognosis, provided that the expression value of IncRNA pairs was 0 or 1 . DE-IncRNA pairs were deemed to be an applicable match when the expression value was more than $20 \%$ of the total pairs. 
The least absolute shrinkage and selection operator (Lasso) regression model ${ }^{[17]}$ was constructed with a P-value of 0.05 . The Lasso regression ran for 1000 cycles, and for each cycle, a random stimulation was set up 1000 times. Next, the frequency of each pair in the 1000-time-repeated Lasso regression model was recorded, and pairs with frequencies more than 100 times were selected for Cox proportional hazard regression analysis, as well as for the construction of the model. The AUC value of each model was calculated and plotted as a curve. If the curve reached the highest point, indicating the maximum AUC value, the calculation procedure was terminated, and the model was considered the optimal candidate. The 1-, 3-, and 5-y ROC curves of the model were plotted. The following formula was then established and applied to all clinical samples. The maximum inflection point was confirmed as the optimal cutoff value to differentiate between high- and low-risk. Ultimately, we conducted a Kaplan-Meier analysis to validate the accuracy of the risk model using the R, and the packages ${ }^{[18]}$ used were "survival", "glmnet", "pbapply", "survivaIROC", "survminer", and "heatmap". Chi-square test was used to analyze the relationship between the risk model and clinical characteristics, whereas the Wilcoxon test was used to evaluate the risk score differences among different clinical groups.

To analyze the relationship between high- or low-risk and immune-cell characteristics, we applied novel methods to calculate the immune infiltration status among BLCA, including TIMER (http://cistrome.org/TIMER/), CIBERSORT, XCELL, QUANTISEQ, MCPcounter, and EPIC. The Wilcoxon signed-rank test was then applied to calculate the differences in infiltrating immune cells between the high- and low-risk groups. Subsequently, the relationship between the risk score values and the immuneinfiltrated cells was evaluated using Spearman's correlation analysis. The significance cutoff was set at $\mathrm{P}<0.05$. The $\mathrm{R}$ package "ggplot2" was used in the process.

In this study, we calculated the half-maximal inhibitory concentration (IC50) of common chemotherapeutic drugs among patients with bladder cancer in the TCGA-BLCA project. The difference between the high- and low-risk groups was determined using the Wilcoxon test, and results were obtained using the packages "pRRophetic" and "ggplot2" in R.

\section{Results}

\section{Identification of DE-ir-IncRNAs and construction of two DE- ir-IncRNA pairs}

A flow chart of the study is shown in Fig. 1. First, we identified the raw data for bladder cancer from the TCGA-BLCA project, which included 19 normal and 411 tumor samples. Then, we annotated the transcriptome based on the gene transfer format (GTF) files, which were downloaded from the Ensembl database, and detected 1269 ir-IncRNAs (Table S1). Among these, 109 were identified as DE-ir-IncRNAs, of which 14 were downregulated, whereas 95 were upregulated (Fig. 2A). Ultimately, we constructed a 0 or 1 matrix to generate DE-ir-IncRNA pairs. In total, we observed that 4896 pairs were constructed, 251 pairs were identified using univariate analysis, and 49 DE-ir-IncRNA pairs were verified by Lasso regression 
model analysis. We established a multi-Cox regression model including 21 DE-IncRNA pairs using the forward method (Fig. 2B).

Establishing a risk assessment and evaluating the relationship between the risk assessment model and prognosis of patients with BLCA

Subsequently, we calculated the areas under the curve (AUCs) for each receiver operating characteristic (ROC) curve for 21 DE-IncRNA pairs (Fig. 3A) and detected the optimal cutoff value, which referred to 1483 using the Akaike information criterion ${ }^{[19]}$ (AIC) values (Fig. 3B). Based on the cutoff point, we classified the patients into high- and low-risk groups. To validate the cutoff value, we delineated the 1-, 3-, and 5-y ROC curves, the AUC values of which were over 0.80, (Fig. 3C), and outlined the 5-y ROC curves with other clinical characteristics (Fig. 3D).

\section{Evaluating the relationship between the risk assessment model and clinical characteristics}

Based on the cutoff value previously detected, 156 patients were categorized into the high-risk group and 244 into the low-risk group. Risk scores and survival of each patient are shown in Fig. 4A and Fig. 4B, confirming that the clinical prognosis of the low-risk group was superior to that of the high-risk group. Moreover, we observed that patients in the low-risk group had longer survival than those in the other group according to analysis using the Kaplan-Meier method $(P<0.0001)$ (Fig. 4C). Next, we conducted chi-square tests to elucidate the relationship between the risk of bladder cancer and clinical characteristics. The ribbon chart and latter diagrams established using the Wilcoxon signed-rank test showed that age (Fig. 5B), grade (Fig. 5C), and stage (Fig. 5D) were significantly associated with the risk group $(P<0.001)$. Then, we demonstrated that age $(P<0.01, H R=1.026,95 \% C l[1.009-1.042])$, stage $(P$ $<0.001, \mathrm{HR}=1.564,95 \% \mathrm{Cl}[1.280-1.912])$, and risk score $(\mathrm{P}<0.001, \mathrm{HR}=1.154,95 \% \mathrm{Cl}$ [1.126-1.182] were statistically significant as indicated by univariate Cox regression model analysis (Fig. 5E), and further verified by multivariate Cox regression analysis.

\section{Assessment Of Tumor-infiltrating Immune Cells With The Risk Model}

After establishing and verifying the risk model, we investigated whether the model was relevant to the tumor immune microenvironment. The high-risk group was more significantly associated with tumorinfiltrating immune cells such as macrophages, neutrophils, and CD8 + T-cells and negatively associated with myeloid dendritic cells and CD4 + T-cells, as verified using the Wilcoxon signed-rank test (Fig. 6A). As ICls have been used to treat bladder cancer in clinical practice, we investigated whether the risk model could include ICl-related biomarkers. We found that high-risk scores were positively correlated with the high expression of discoidin domain receptor tyrosine kinase 2 (DDR2) $(P<0.05$, Fig. 6C) and hepatitis A virus cellular receptor 2 (HAVCR2) $(P<0.001$, Fig. 6D), whereas lymphocyte activating 3 (LAG3) $(P>0.05$, Fig. 6E), and cytotoxic T-lymphocyte associated protein 4 (CTLA4) $(P>0.05$, Fig. $6 \mathrm{~A})$ showed no significant differences. 


\section{Assessment of the relationship between the risk model and clinical chemotherapeutics}

In addition to the aforementioned immunotherapy, we aimed to identify the relationships between the risk model and the efficacy of common chemotherapeutics in treating bladder cancer from the TCGA-BLCA project. Our results showed that a high-risk score was associated with a lower IC50 of chemotherapeutics such as cisplatin $(P=0.00021$, Fig. $7 A)$, docetaxel $(P<0.0001$, Fig. 7B), and paclitaxel $(P<0.0047$, Fig. 7C). In contrast, we found that it was associated with a higher IC50 for metformin $(P<0.001$, Fig. 7D) and methotrexate $(P<0.001$, Fig. 7E). Collectively, these results indicated the predictive value of the risk model.

\section{Discussion}

Previous studies have already reported both coding mRNAs and non-coding RNAs to predict the prognosis of patients with malignant tumors, but almost all these prognosis models were established based on the expression levels of coding genes ${ }^{[20,21]}$. In the present study, we established a prognostic risk model by pairing immune-related genes for the first time and constructed a risk model with two IncRNA pairs, without adopting their exact expression value. First, we downloaded raw data of IncRNAs from TCGA-BLCA, acquired DE-IncRNAs, conducted a coexpression analysis to detect DE-ir-IncRNAs, and validated the obtained DE-ir-IncRNA-pairs using a 0-or-1 matrix. Second, we applied a modified Lasso penalized regression model, including procedures of the cross, multiple repetitions of validation, and random stimulation to determine DE-ir-IncRNA pairs. Third, we delineated ROC curves and calculated the AUC values to acquire the optimized model. In addition, we calculated the AIC value of each point on the AUC to detect the top cutoff to differentiate the high-or low-risk groups among patients with bladder cancer. Finally, we verified this novel risk model in different situations, including clinical characteristics, various immune cells, chemotherapeutics, and immunotherapeutic biomarkers.

Various studies have established a signature based on the expression of several IncRNAs for predicting the survival of patients with bladder cancer. It is well known that high expression of IncRNAs generally has crucial biological functions. Lian et al. ${ }^{[22]}$ established an 8-IncRNA signature containing APCDD1LAS1, FAM225B, LINC00626, LINC00958, LOC100996694, LOC441601, LOC101928111, and ZSWIM8-AS1 as candidate prognostic markers for bladder cancer. Based on the signature, they constructed a risk model and validated its prognostic value. However, all these studies were based on the exact expression of IncRNAs. In the study, we detected the DE-ir-IncRNAs based on the immure-related genes and constructed conspicuous ir-IncRNA pairs. Then, we detected pairs using sorting parameters instead of examining the specific expression value of each IncRNA. Some of the DE-ir-IncRNAs detected in this study, such as TRPM2-AS, LINC01605, AC104041.1, and UCA1, have been confirmed to play significant roles in the progression of bladder cancer. Avgeris et al. ${ }^{[23]}$ detected that downregulation of UCA1 was correlated with a higher risk of short-term relapse in bladder cancer. Tian et al. ${ }^{[24]}$ reported that TRPM2-AS promoted bladder cancer by targeting miR-22-3p and regulating the expression of GINS2 mRNA. Qin et al.

${ }^{[25]}$ found that high LINC01605 expression promoted the progression of bladder cancer by upregulating 
MMP9. In this study, we established a Cox regression model that was confirmed using the Lasso regression model to enhance the efficacy of the predicted value of the prognostic model. Thus, we calculated every AUC value to detect the maximum value for a risk model and to obtain the cutoff value for the risk model with the AIC point. In addition, we analyzed the therapeutic effect of chemotherapeutics for BLCA, tumor environment immune infiltration, and immunotherapy-related markers.

Previous preclinical studies have confirmed that increased infiltration of CD $4+$ or CD $8+$ immune cells ${ }^{[26-}$ ${ }^{28]}$ leads to a better response to immune checkpoint inhibitors. In the present study, we further used various methods, including CIBERSORT, XCELL, CIBERSORT-ABS, QUANTISEQ, MCPcounter, EPIC, and TIMER to estimate the tumor-infiltrating cells, as well as their association with risk scores. Our results showed that CD4 + T-cells, monocytes, macrophages, cancer-associated fibroblasts, and myeloid dendritic cells were enriched in the high-risk group. Previous studies have demonstrated that LINC00665 enhanced the efficacy of immunotherapy in bladder cancer ${ }^{[29-31]}$. In this study, as signatures were not shown to be positively correlated with checkpoint-related biomarkers, novel biomarkers, other than signatures, should be detected and validated. Our risk model implied that the high-risk group was more sensitive to methotrexate and metformin, whereas the low-risk group was more sensitive to cisplatin, docetaxel, and paclitaxel.

There were some limitations to our study. First, the raw data downloaded from the TCGA database were relatively insufficient for initial analysis. Second, external validation was necessary to verify the risk model established in this study. To remedy this difference, we screened IncRNA pairs initially using the 0or-1 matrix, which was optimal in this study. We aim to gather more clinical samples for further verification; however, this collection will take time. In summary, we detected a novel model comprising irIncRNAs, which did not require the exact expression level of IncRNAs and would help clinicians identify patients who might benefit from immunotherapy.

\section{Abbreviations}

AIC, Akaike information criterion; AUC, area under the curve; BCG, Bacillus Calmette-Guérin; BC, bladder cancer; DE-IncRNAs, differentially expressed IncRNAs; FDR, false discovery rate; FC, fold change; FPKM, fragments per kilobase million; ICls, immune checkpoint inhibitors; ir-IncRNA, immune-related IncRNA; IC50, inhibitory concentration; IncRNAs, long non-coding RNAs; MIBC, muscle-invasive bladder cancer; NMIBC, non-muscle-invasive bladder cancer; ROC, receiver operating characteristic; UCA1, urothelial carcinoma-associated IncRNA.

\section{Declarations}

\section{Ethics approval and consent to participate}

All analyses were based on online database, thus no ethical approval and patient consent are required

\section{Consent for publication}


None

\section{Availability of data and materials}

All the data used in this study can be obtained from online database.

\section{Competing interests}

The author(s) declare no potential conflicts of interest concerning the research, authorship, and/or publication of this article.

\section{Funding}

The author(s) disclose receipt of the following financial support for the research, authorship, and/or publication of this article: Financial support was provided by Jiaxing Science and Technology Bureau (No.2021AD3017, No.2020AD30084) and Medical and Health Science and Technology Project of Zhejiang Province (No.2021KY354).

\section{Authors' contributions}

Z. Gao was responsible for the design. P. Fu and FM. Fei provided the administrative support. T. Sun and TH. Zhu were for the collection and assembly of data. LH. Yuan, DJ. Wu, WW. Zheng and Z. Gao were responsible for manuscript writing. All the authors approved the final manuscript. Our study did not require an ethical board approval because it did not contain human or animal trials.

\section{Acknowledgments}

Thanks for the support from the Dean of Jiaxing Second hospital (G. Chen)

\section{Authors' information}

1 Department of Clinical Oncology, The Second Affiliated Hospital of Jiaxing University, Jiaxing, China. 2 Department of General Medicine, Nanhu District Central Hospital of Jiaxing, Jiaxing, China. 3 Department of Orthopedic Oncology, The Second Affiliated Hospital of Jiaxing University, Jiaxing, China. 4 Hospice care center of Jiaxing second hospital.

\section{References}

1. Afonso J, Santos LL, Longatto-Filho A, Baltazar F. Competitive glucose metabolism as a target to boost bladder cancer immunotherapy. Nature reviews Urology. 2020;17:77-106.

2. Autio KA, Boni V, Humphrey RW, Naing A. Probody Therapeutics: An Emerging Class of Therapies Designed to Enhance On-Target Effects with Reduced Off-Tumor Toxicity for Use in ImmunoOncology. Clinical cancer research: an official journal of the American Association for Cancer Research. 2020;26:984-9. 
3. Benitez JC, Remon J, Besse B. Current Panorama and Challenges for Neoadjuvant Cancer Immunotherapy. Clinical cancer research: an official journal of the American Association for Cancer Research. 2020;26:5068-77.

4. Fradet Y, Bellmunt J, Vaughn DJ, Lee JL, Fong L, Vogelzang NJ, et al. Randomized phase III KEYNOTE-045 trial of pembrolizumab versus paclitaxel, docetaxel, or vinflunine in recurrent advanced urothelial cancer: results of $>2$ years of follow-up. Ann Oncol. 2019;30:970-6.

5. Snyder A, Nathanson T, Funt SA, Ahuja A, Buros Novik J, Hellmann MD, et al. Contribution of systemic and somatic factors to clinical response and resistance to PD-L1 blockade in urothelial cancer: An exploratory multi-omic analysis. PLoS Med. 2017;14:e1002309.

6. Jacqueline Vuky AVB, Bellmunt J, Fang X, Plimack ER, Castellano D, Godwin JL, Powles T, Bajorin D, Frenkl TL, Peter H, O'Donnell NM, Hahn BH, Moreno, ; Petros Grivas MJS. Ronald de Wit.: Long-Term Outcomes in KEYNOTE-052: Phase II Study Investigating First-Line Pembrolizumab in CisplatinIneligible Patients With Locally Advanced or Metastatic Urothelial Cancer. J Clin Oncol. 2020;38:2658-66.

7. Tran L, Xiao JF, Agarwal N, Duex JE, Theodorescu D. Advances in bladder cancer biology and therapy. Nat Rev Cancer. 2021;21:104-21.

8. Linxweiler J, Junker K. Extracellular vesicles in urological malignancies: an update. Nature reviews Urology. 2020;17:11-27.

9. Pan J, Li X, Wu W, Xue M, Hou H, Zhai W, et al. Long non-coding RNA UCA1 promotes cisplatin/gemcitabine resistance through CREB modulating miR-196a-5p in bladder cancer cells. Cancer Lett. 2016;382:64-76.

10. Chen C, He W, Huang J, Wang B, Li H, Cai Q, et al. LNMAT1 promotes lymphatic metastasis of bladder cancer via CCL2 dependent macrophage recruitment. Nature communications. 2018;9:3826.

11. Chen $\mathrm{C}$, Luo Y, He W, Zhao Y, Kong Y, Liu H, et al. Exosomal long noncoding RNA LNMAT2 promotes lymphatic metastasis in bladder cancer. J Clin Investig. 2020;130:404-21.

12. Chen X, Xie R, Gu P, Huang M, Han J, Dong W, et al. Long Noncoding RNA LBCS Inhibits Self-Renewal and Chemoresistance of Bladder Cancer Stem Cells through Epigenetic Silencing of SOX2. Clinical cancer research: an official journal of the American Association for Cancer Research. 2019;25:1389403.

13. Zhan Y, Chen Z, He S, Gong Y, He A, Li Y, et al. Long non-coding RNA SOX2OT promotes the stemness phenotype of bladder cancer cells by modulating SOX2. Mol Cancer. 2020;19:25.

14. Zhan Y, Du L, Wang L, Jiang X, Zhang S, Li J, et al. Expression signatures of exosomal long noncoding RNAs in urine serve as novel non-invasive biomarkers for diagnosis and recurrence prediction of bladder cancer. Mol Cancer. 2018;17:142.

15. Zheng R, Du M, Wang X, Xu W, Liang J, Wang W, et al. Exosome-transmitted long non-coding RNA PTENP1 suppresses bladder cancer progression. Mol Cancer. 2018;17:143.

16. Wu Y, Zhang L, He S, Guan B, He A, Yang K, et al. Identification of immune-related LncRNA for predicting prognosis and immunotherapeutic response in bladder cancer. Aging. 2020;12:23306-25. 
17. TIBSHIRANI R: THE LASSO METHOD FOR VARIABLE SELECTION IN THE COX MODEL. STATISTICS IN MEDICINE. 1997;16:385-95.

18. Robert C, Gentleman VJC, Douglas M, Bates B, Bolstad M, Dettling S, Dudoit B, Ellis L, Gautier Y, Ge J, Gentry K, Hornik T, Hothorn W, Huber S, lacus R, Irizarry F, Leisch C, Li M, Maechler AJ, Rossini. Gunther Sawitzki, Colin Smith, Gordon Smyth, Luke Tierney, Jean YH Yang and Jianhua Zhang: Bioconductor: open software development for computational biology and bioinformatics. Genome Biol. 2004;5:80-6.

19. Posada D, Buckley TR. Model selection and model averaging in phylogenetics: advantages of akaike information criterion and bayesian approaches over likelihood ratio tests. Syst Biol. 2004;53:793808.

20. Robertson AG, Kim J, Al-Ahmadie H, Bellmunt J, Guo G, Cherniack AD, et al. Comprehensive Molecular Characterization of Muscle-Invasive Bladder Cancer. Cell. 2017;171:540-56. e25.

21. Zhou M, Zhang Z, Bao S, Hou P, Yan C, Su J, et al: Computational recognition of IncRNA signature of tumor-infiltrating $B$ lymphocytes with potential implications in prognosis and immunotherapy of bladder cancer. Brief Bioinform 2020.

22. Penghu Lian QW, Zhao Y, Chen C, Sun X, Li H, Deng J, Zhang H, Ji Z, Zhang X. Qichao Huang: An eight-long non-coding RNA signature as a candidate prognostic biomarker for bladder cancer. Aging, 11:6930-40.

23. Avgeris M, Tsilimantou A, Levis PK, Rampias T, Papadimitriou MA, Panoutsopoulou K, et al. Unraveling UCA1 IncRNA prognostic utility in urothelial bladder cancer. Carcinogenesis. 2019;40:965-74.

24. Tian Y, Guan Y, Su Y, Yang T, Yu H. TRPM2-AS Promotes Bladder Cancer by Targeting miR-22-3p and Regulating GINS2 mRNA Expression. Onco Targets Ther. 2021;14:1219-37.

25. Qin Z, Wang Y, Tang J, Zhang L, Li R, Xue J, et al: High LINC01605 expression predicts poor prognosis and promotes tumor progression via up-regulation of MMP9 in bladder cancer. Biosci Rep 2018, 38.

26. Elizabeth Pluhara G CAP, and Olin MR. CD8 + T Cell-Independent Immune-Mediated Mechanisms of AntiTumor Activity. Crit Rev Immunol, 35:153-72.

27. Ostroumov D, Fekete-Drimusz N, Saborowski M, Kuhnel F, Woller N. CD4 and CD8 T lymphocyte interplay in controlling tumor growth. Cell Mol Life Sci. 2018;75:689-713.

28. Tay RE, Richardson EK, Toh HC. Revisiting the role of CD4(+) T cells in cancer immunotherapy-new insights into old paradigms. Cancer Gene Ther. 2021;28:5-17.

29. Zhang Y, Liu Q, Liao Q. Long noncoding RNA: a dazzling dancer in tumor immune microenvironment. J Exp Clin Cancer Res. 2020;39:231.

30. Cong Z, Diao Y, Xu Y, Li X, Jiang Z, Shao C, et al. Long non-coding RNA linc00665 promotes lung adenocarcinoma progression and functions as ceRNA to regulate AKR1B10-ERK signaling by sponging miR-98. Cell Death Dis. 2019;10:84. 
31. Yang D, Feng W, Zhuang Y, Liu J, Feng Z, Xu T, et al. Long non-coding RNA linc00665 inhibits CDKN1C expression by binding to EZH2 and affects cisplatin sensitivity of NSCLC cells. Mol Ther Nucleic Acids. 2021;23:1053-65.

\section{Figures}

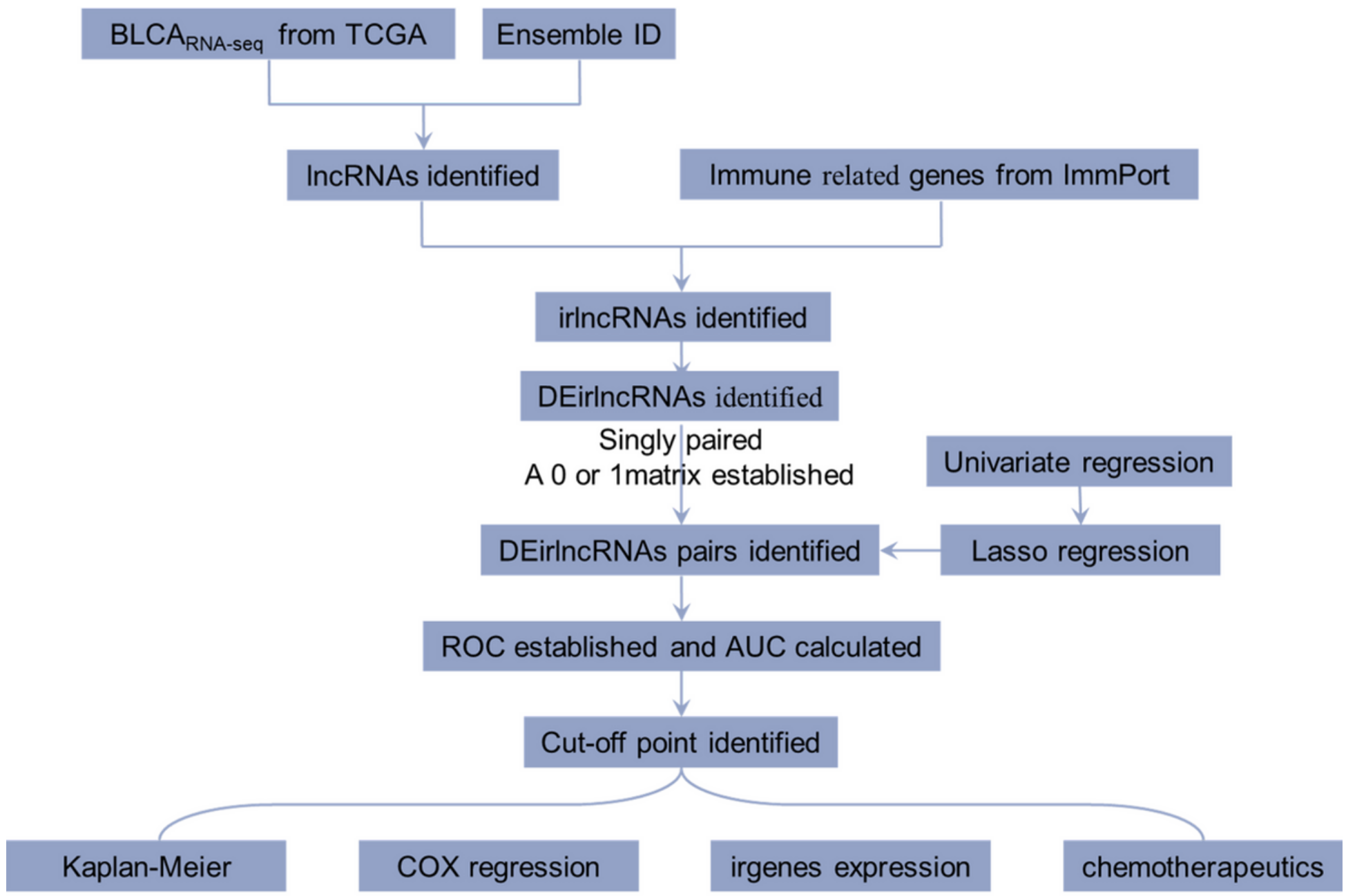

\section{Figure 1}

Detailed flow chart of the study. 

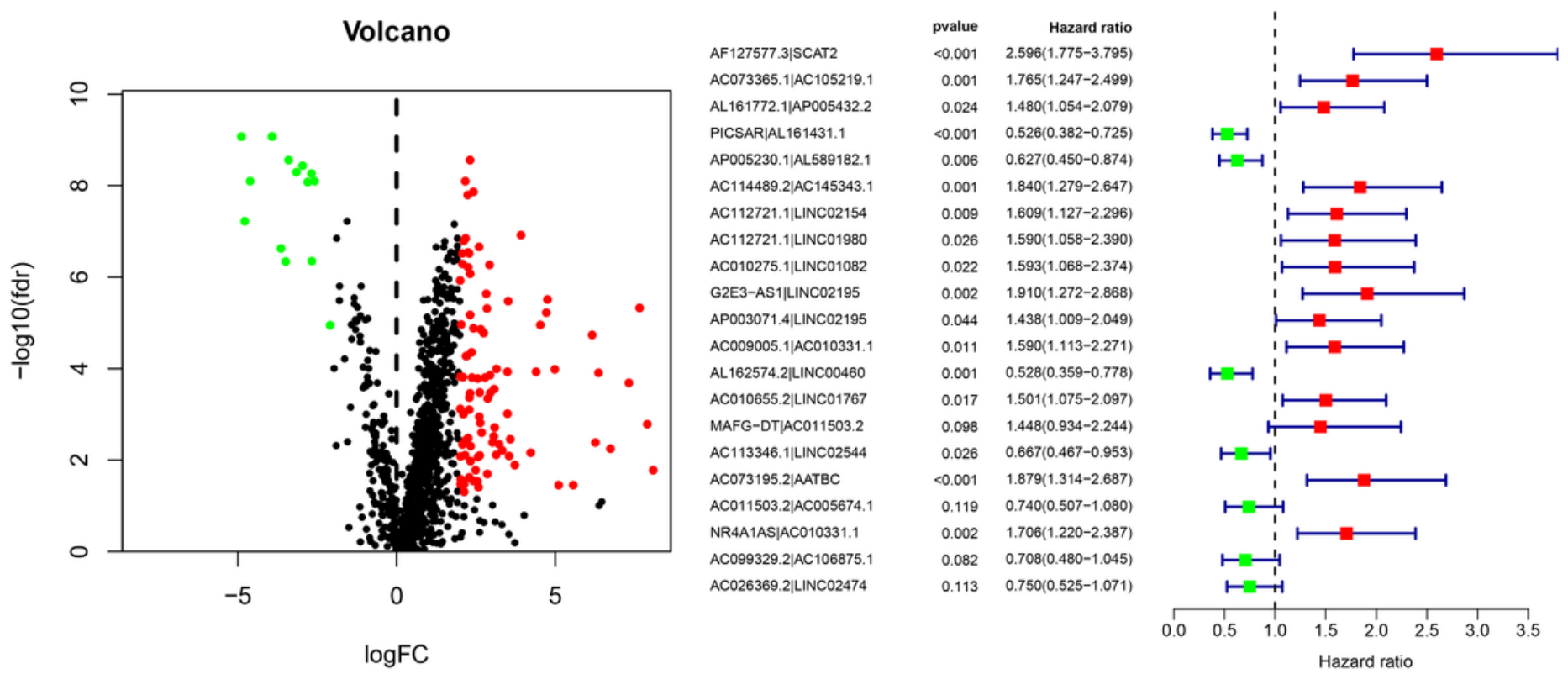

Figure 2

Detection of DEirlncRNAs(A) and identification of 21 DElncRNAs by COX regression model(B). 


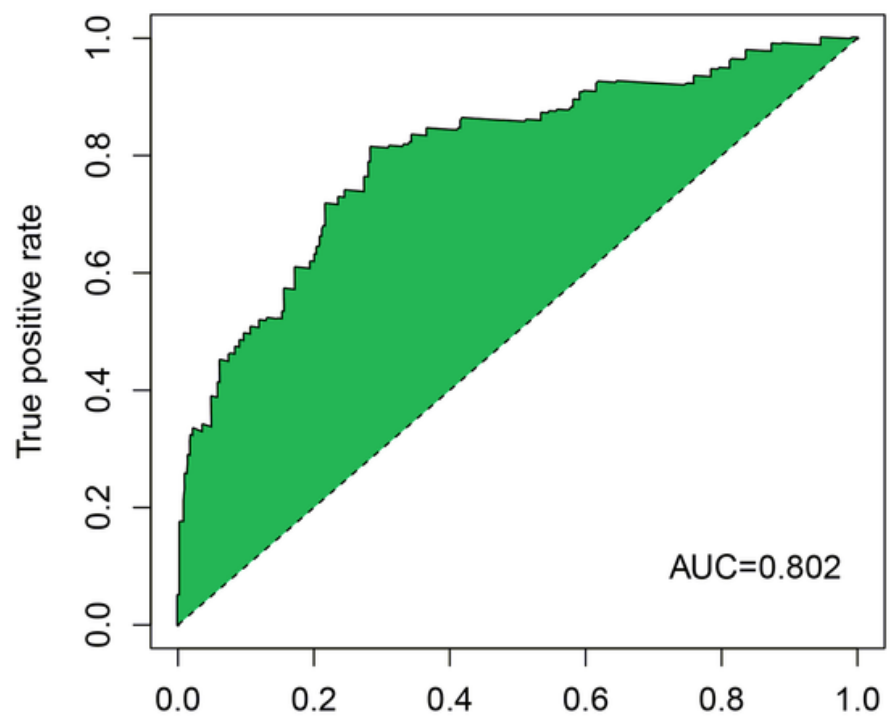

False positive rate

C

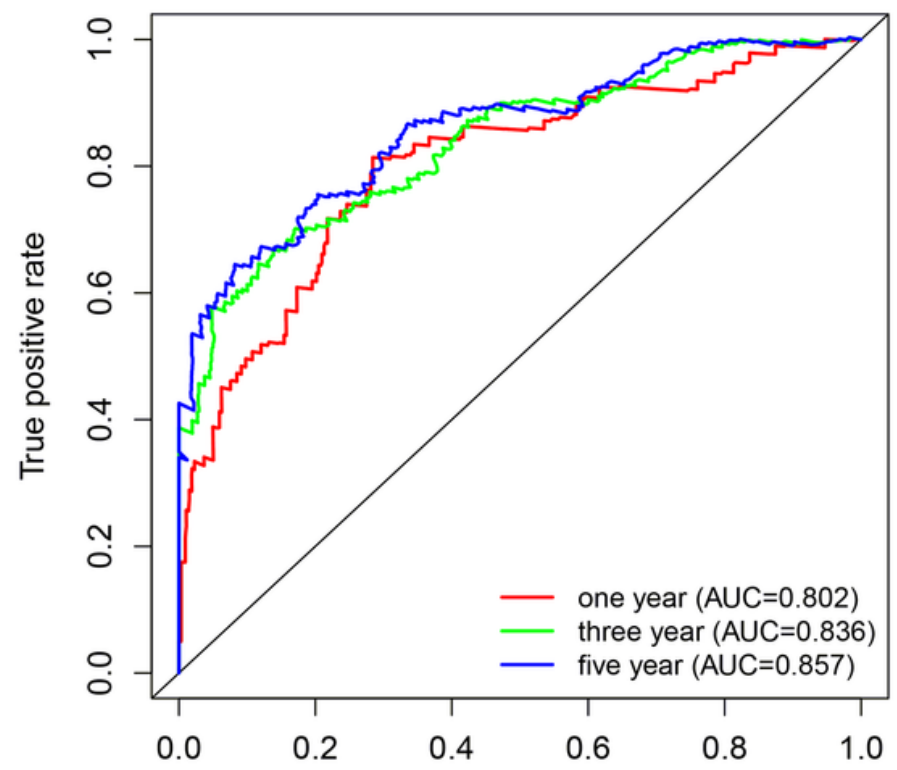

False positive rate

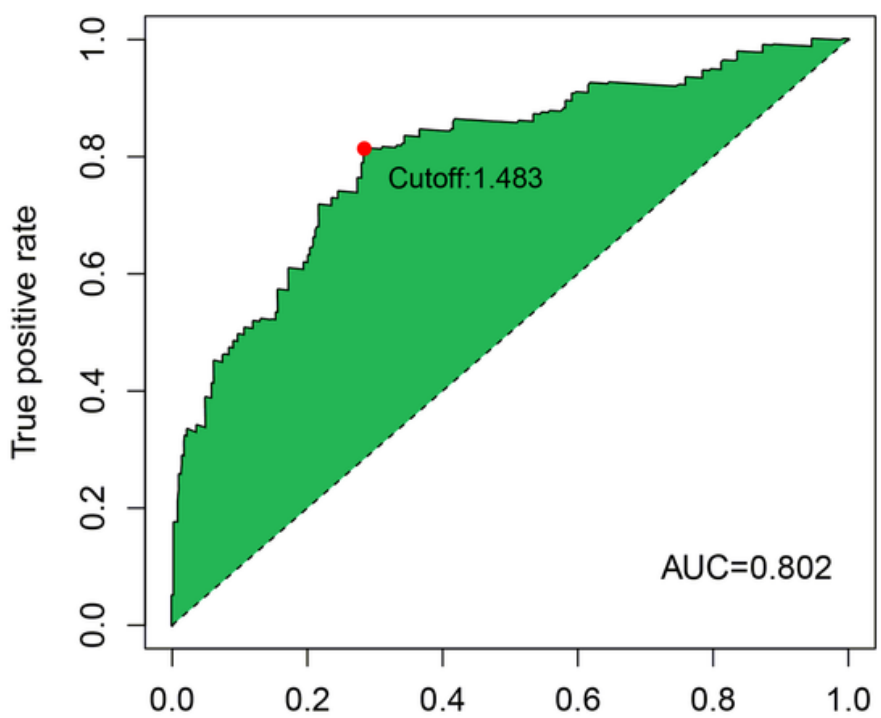

False positive rate

D

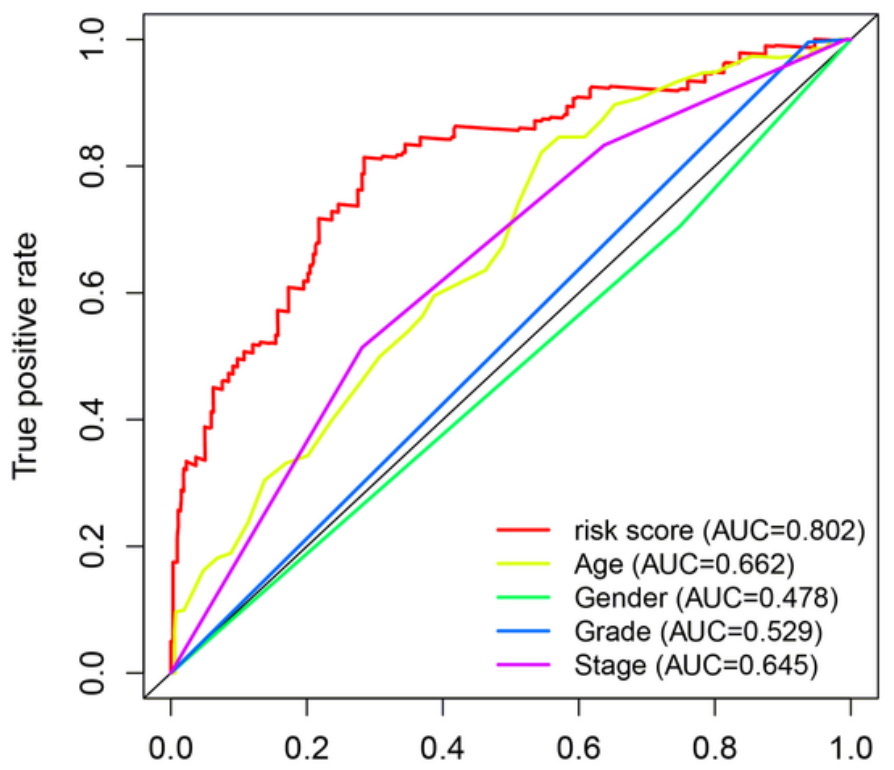

False positive rate

\section{Figure 3}

The model consisting 21 DEirlncRNA pairs related to the optimal AUC(A). All AUC values of the model were over $0.80(B)$. AUC of 1-year ROC curves was compared with common clinical characteristics(C). (E) RiskScore for 430 patients with BC, and cut-off point showed in this figure was obtained by the AIC. 

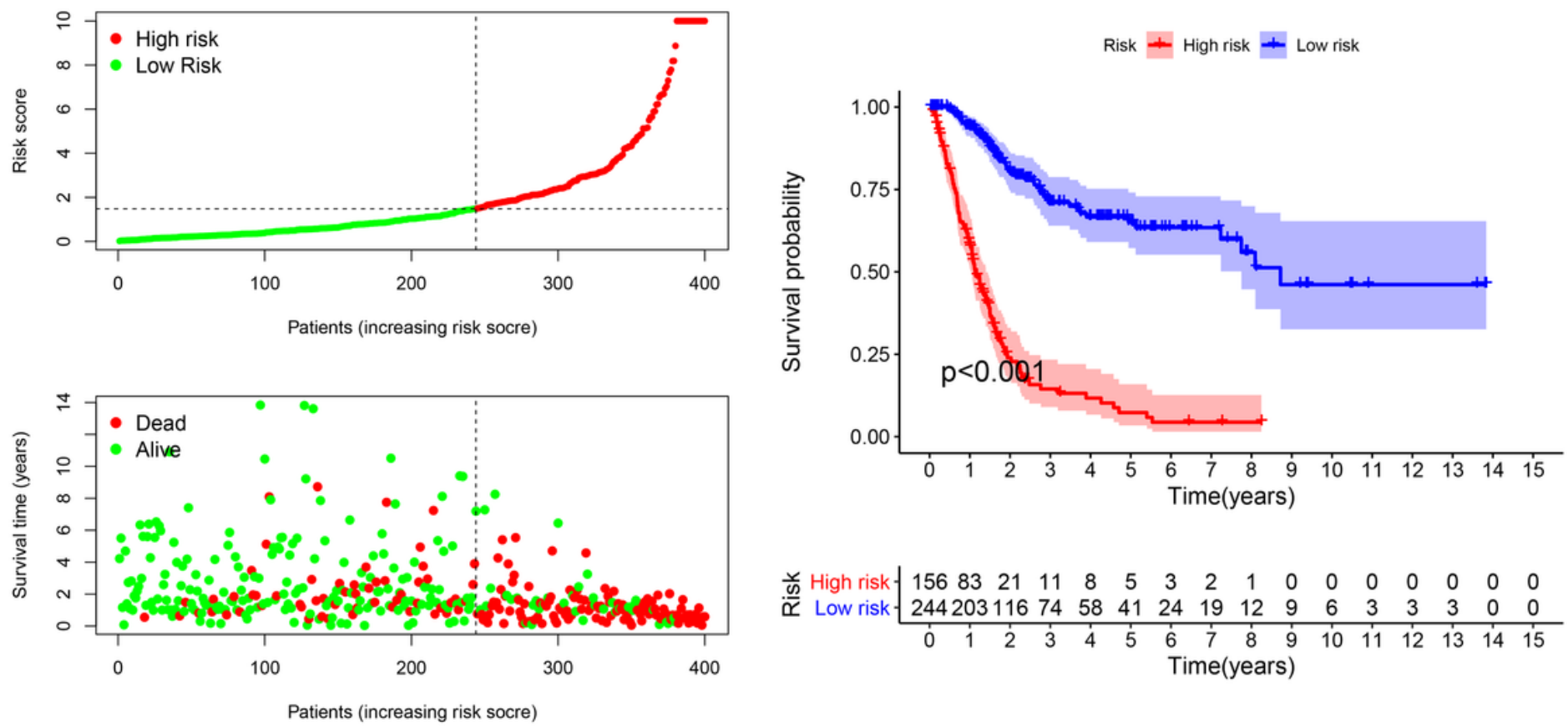

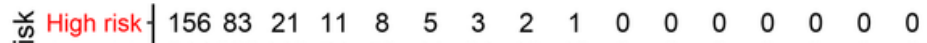

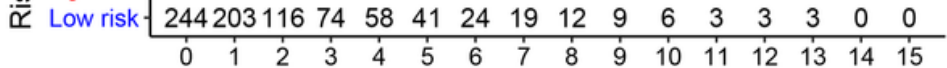
Time(years)

\section{Figure 4}

The relationship between the model and prognosis was assessed. The risk score and survival outcome of each case are visualized(A, B). Survival curves of different groups were plotted by Kaplan-Meier(C). 
A
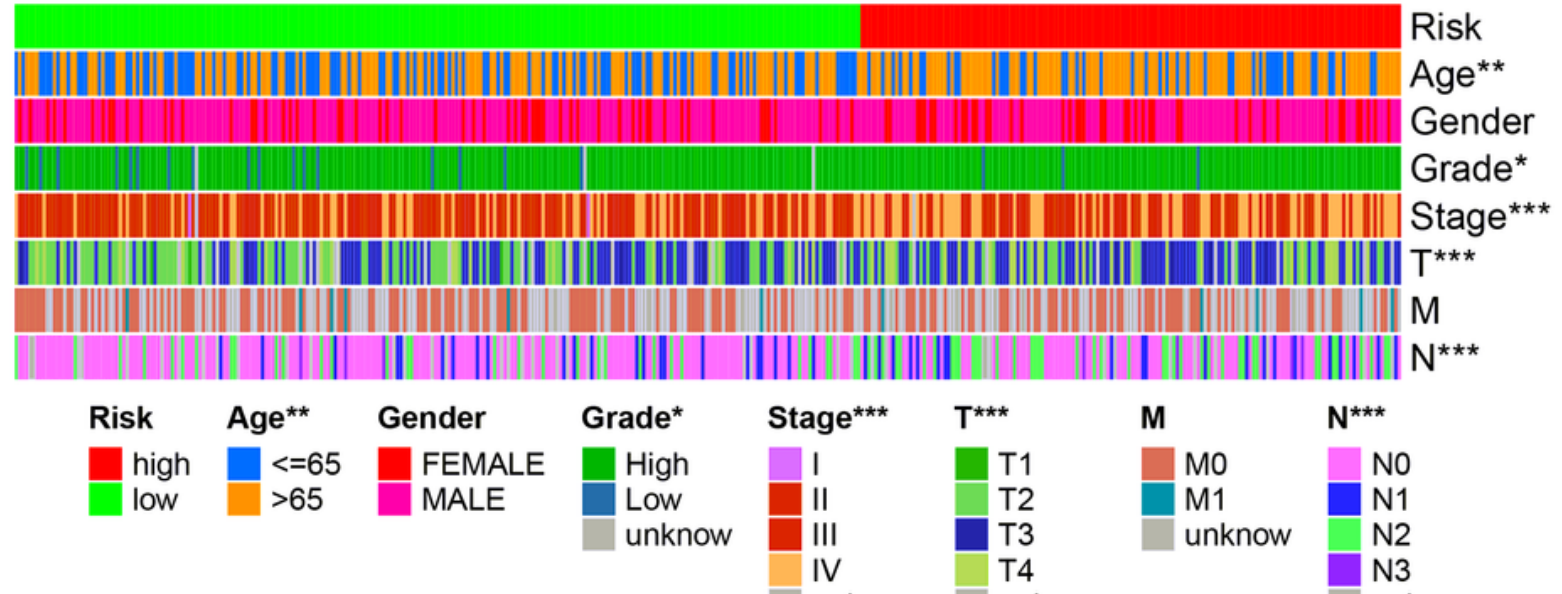

B

C unknow unknow unknow
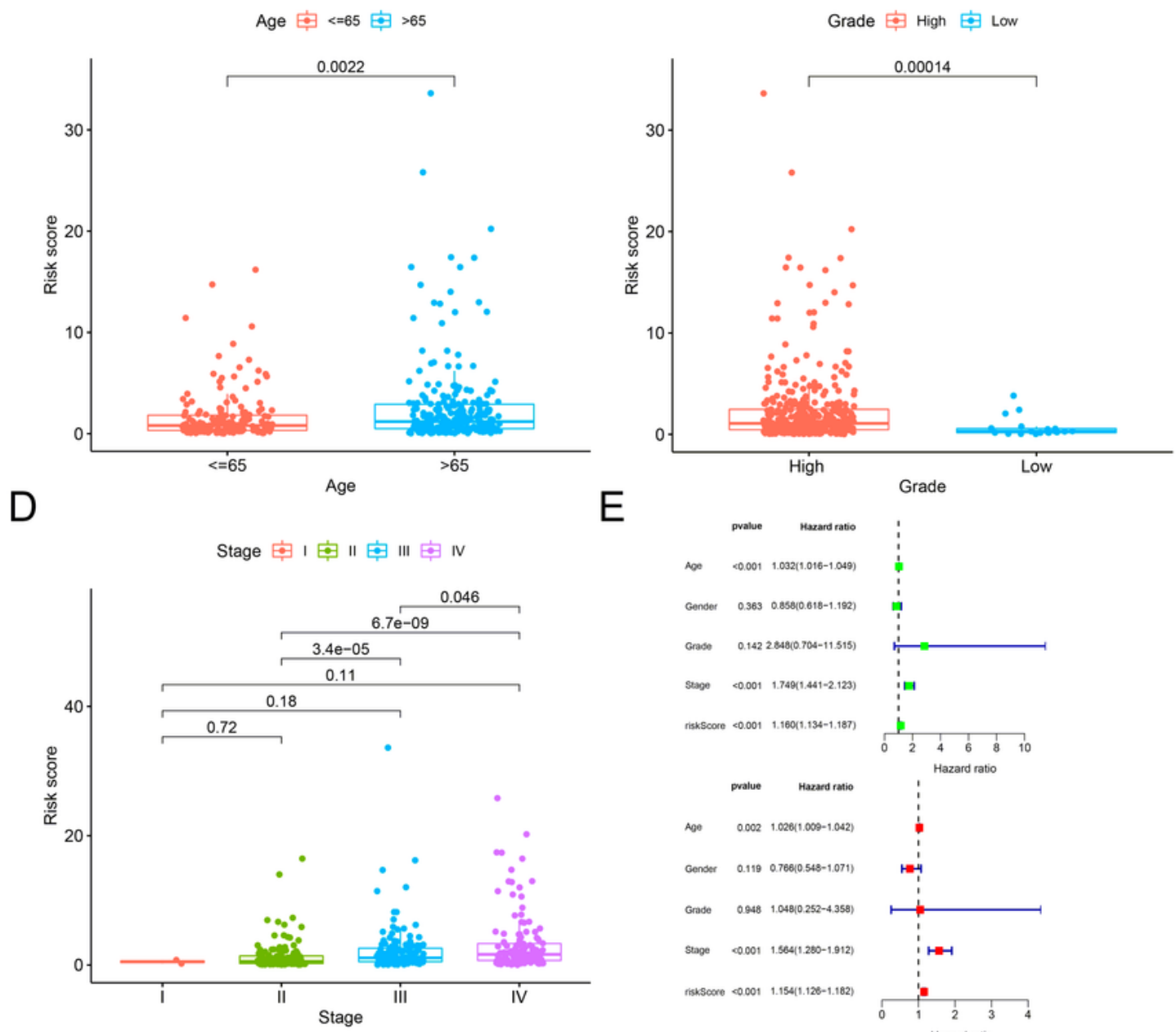

\section{Figure 5}

The plotted strip chart(A) and the scatter diagrams showed that Age(B), Grade(C), and Stage(D) were significantly related to the riskScore. The univariate Cox regression model analysis showed that stage $(p$ $<0.001)$, age $(p<0.001)$, and riskScore $(p<0.001)(E)$ were statistically different, which were verified by multi-Cox regression model analysis. 
A

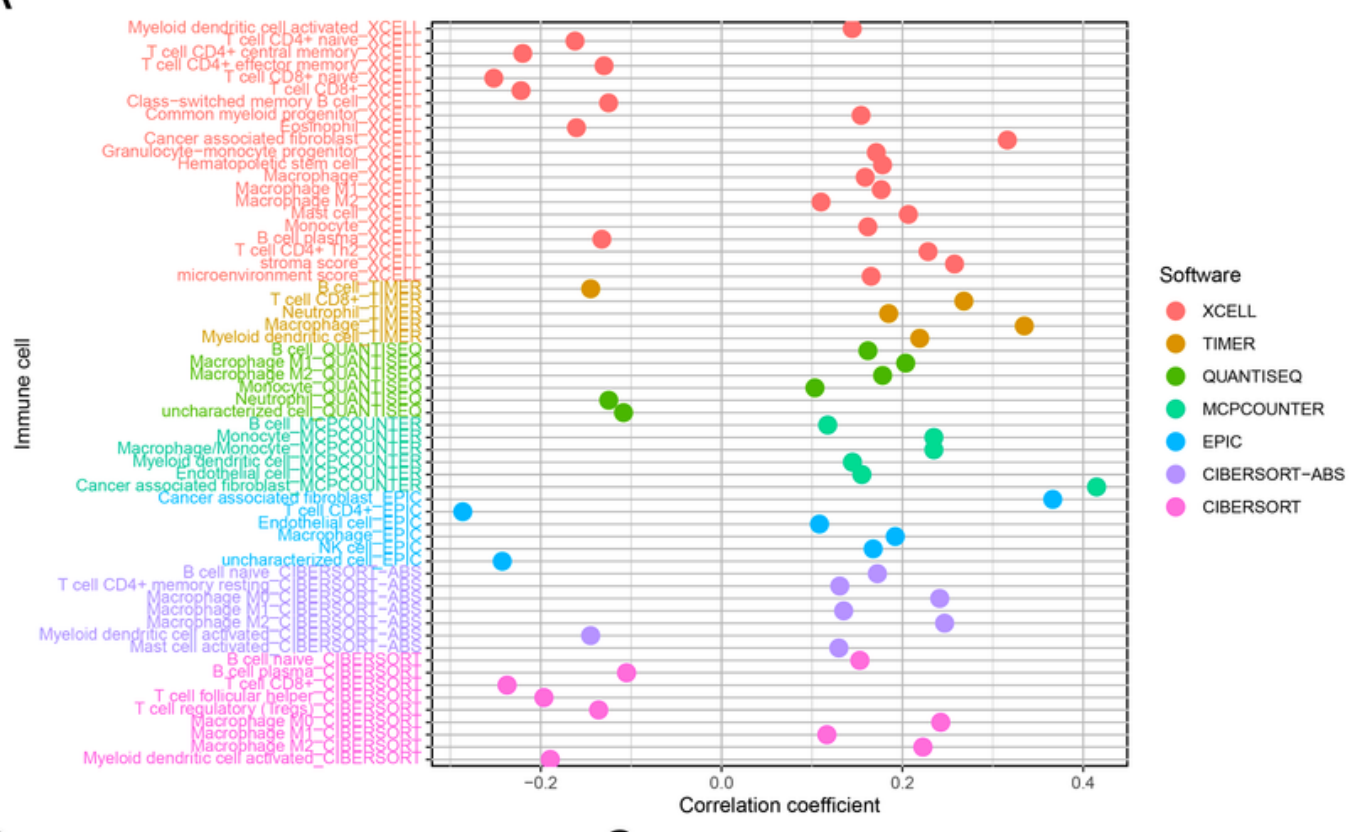

B
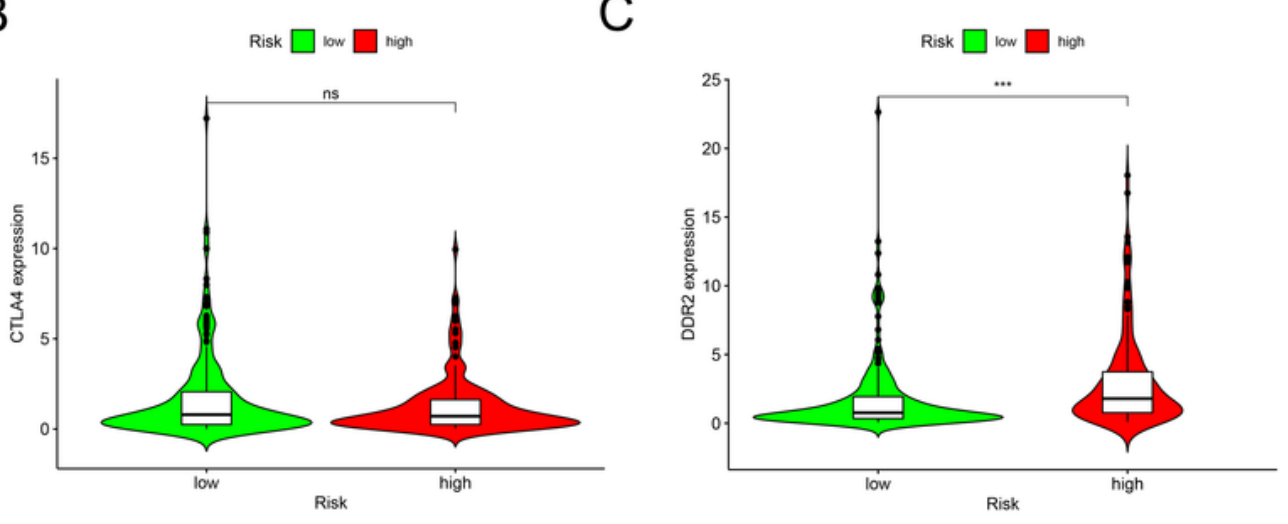

D

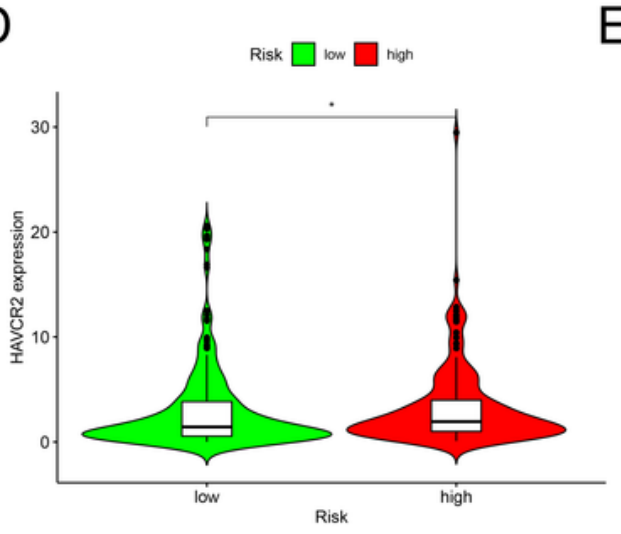

E

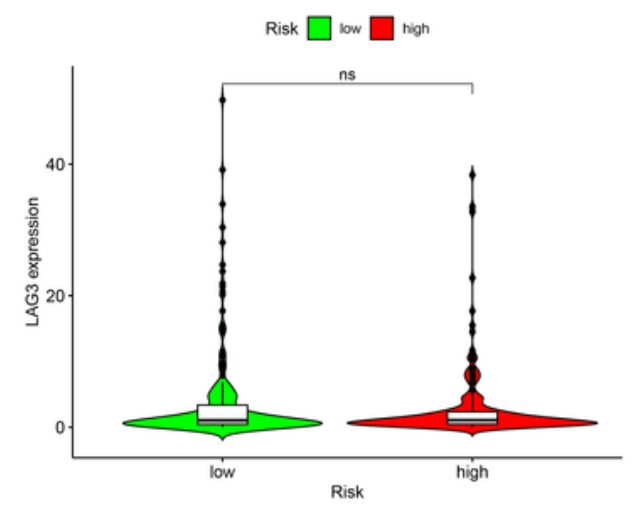

\section{Figure 6}

Spearman correlation analysis was used to detect the infiltration of different immune cells(A). High risk scores were positively correlated with upregulated expression of DDR2(C), HAVCR2(D), whereas LAG3(E), and CTLA4(B) showed no statistical difference in patients with BC. 
A

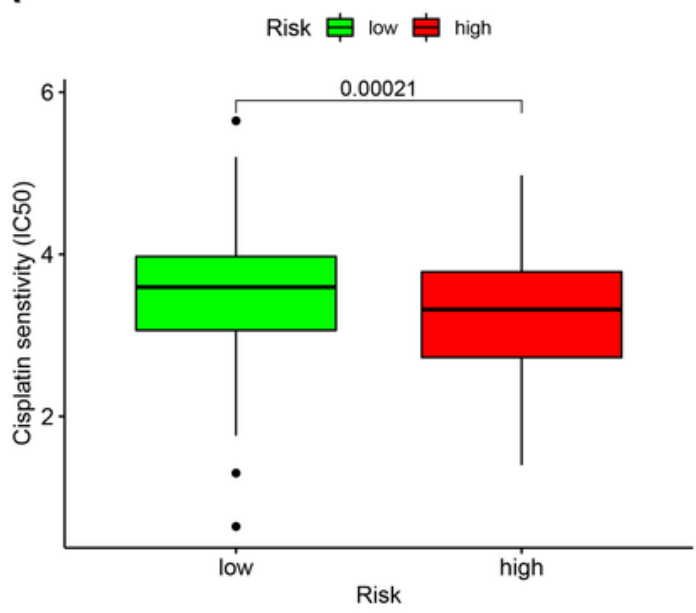

C Risk 追 low 追 high

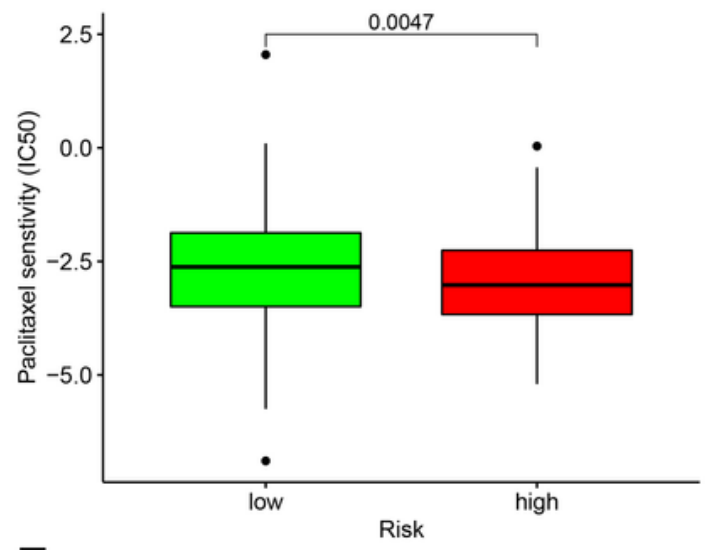

$\mathrm{E}$

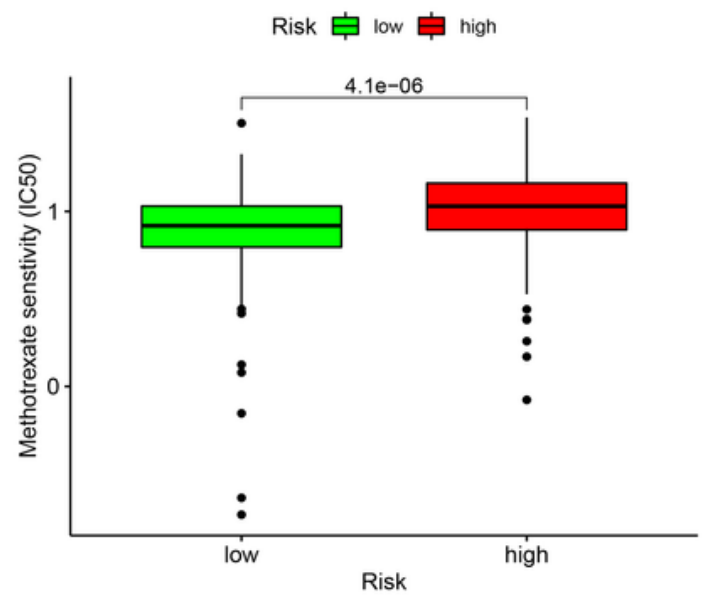

B

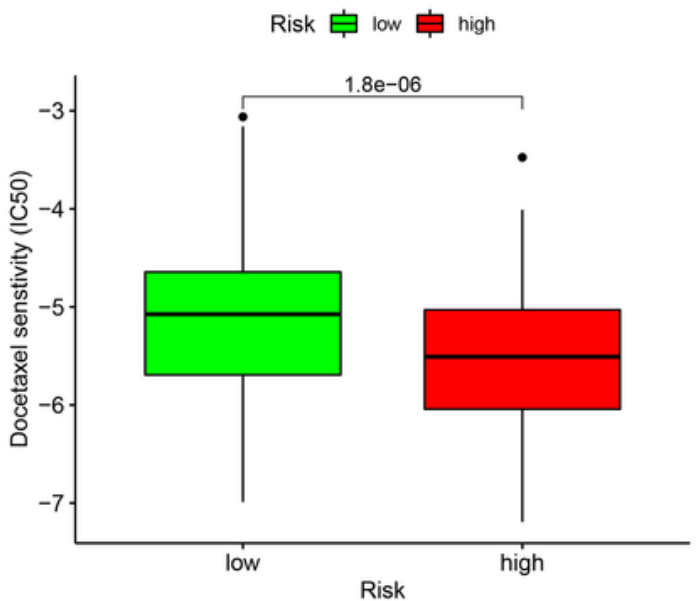

D

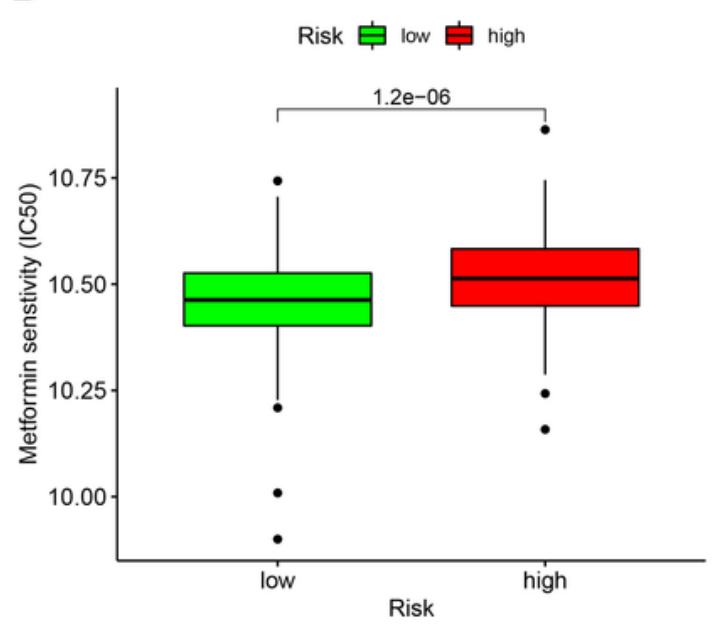

\section{Figure 7}

The model was an established predictor for chemosensitivity, and high risk were related to a lower IC50 for chemotherapeutics such as doxorubicin, paclitaxel, and cisplatin, whereas they were related to a higher IC50 for Metformin and methotrexate. 